\title{
Inextensible flows of spacelike curves according to equiform frame in 4-dimensional Minkowski space $\mathbb{R}_{1}^{4}$
}

\author{
W. M. Mahmoud ${ }^{1}$, Alaa Hassan Noreldeen ${ }^{2}$ \\ ${ }^{1,2}$ Department of Mathematics \\ Aswan University \\ Faculty of Science \\ Aswan, EGYPT
}

\begin{abstract}
In this paper, we study inextensible flows of spacelike curves lying fully on a spacelike surface $\Omega$ according to equiform frame in 4-dimensional Minkowski space $\mathbb{R}_{1}^{4}$. We give necessary and sufficient conditions for this inextensible flows which are expressed as a partial differential equation involving the equiform curvature functions in 4-dimensional Minkowski space $\mathbb{R}_{1}^{4}$. Finally we give an application of inextensible flows of spacelike curves in $\mathbb{R}_{1}^{4}$.
\end{abstract}

Key-Words: - Curvature flows, equiform frame, inextensible, Minkowski space-time.

Received: June 18, 2020. Revised: November 29, 2020. Accepted: December 10, 2020. Published: December 31, 2020.

\section{Introduction}

The flow of a curve or surface is said to be inextensible if, in the former case, the arclength is preserved, and in the latter case, if the intrinsic Curvature is preserved $[10,11,15,18$, 19].

Physically, inextensible curve and surface flows are characterized by the absence of any strain energy induced from the motion $[1,7,8]$. Some relevant studies can be found in [3] and [4].

In this paper, we derive a general formulation for inextensible flows of spacelike curves according to equiform frame in 4-dimensional Minkowski space $\mathbb{R}_{1}^{4}$. In Section 2, we clarify the basic conceptions of 4-dimensional Minkowski space $\mathbb{R}_{1}^{4}$ and give of equiform Fremet frame that will be used during this work. In Section 3, we using the equiform frame to present the necessary and sufficient conditions for the inextensible flow as a partial differential equation involving the equiform curvature functions in 4-dimensional Minkowski space $\mathbb{R}_{1}^{4}$. In Section 4 , we give an application of inextensible flows of spacelike curves in $\mathbb{R}_{1}^{4}$.

\section{Preliminaries}

The 4-dimensional Minkowski space $\mathbb{R}_{1}^{4}$ is the 3- dimensional Euclidean space $\mathbb{R}_{1}^{4}$ provided with the metric

$$
\xi=-d x_{1}^{2}+d x_{2}^{2}+d x_{3}^{2}+d x_{4}^{2}
$$

Where $\left(x_{1}, x_{2}, x_{3}, x_{4}\right)$ is a rectangular coordinate system of $\mathbb{R}_{1}^{4}$. Any arbitrary vector $v \in \mathbb{R}_{1}^{4}$ can have one of three Lorentzian clause depicts; it can be a spacelike, timelike or lightlike if $\mathfrak{F}(v, v)$ is positive, negative or zero respectively. Similarly, any arbitrary curve $\xi=$ $\xi(s)$ can locally spacelike, timelike, or lightlike if $\mathfrak{F}(\dot{\zeta}(s), \dot{\zeta}(s))$ is positive, negative or zero respectively [14].

For any $u, v, w \in \mathbb{R}_{1}^{4}$, the vector product in 4-dimensional Minkowski space $\mathbb{R}_{1}^{4}$ is defined by [17]:

$$
u \wedge v \wedge w=-\left|\begin{array}{cccc}
-e_{1} & e_{2} & e_{3} & e_{4} \\
u_{1} & u_{2} & u_{3} & u_{4} \\
v_{1} & v_{2} & v_{3} & v_{4} \\
w_{1} & w_{2} & w_{3} & w_{4}
\end{array}\right|
$$

Let $\zeta: I \subset \mathbb{R} \rightarrow \mathbb{R}_{1}^{4}$ be a regular spacelike curve parametrized by arc-length in $\mathbb{R}_{1}^{4}$ with timelike second binormal. Then the Frenet formulas along $\zeta$ can be given as $[5,16]$ :

$$
\begin{aligned}
& \left(\begin{array}{c}
\dot{t}(s) \\
\dot{n}(s) \\
\dot{b_{1}}(s) \\
\dot{b_{2}}(s)
\end{array}\right) \\
& =\left(\begin{array}{cccc}
0 & k_{1}(s) & 0 & 0 \\
-k_{1}(s) & 0 & k_{2}(s) & 0 \\
0 & -k_{2}(s) & 0 & k_{3}(s) \\
0 & 0 & k_{3}(s) & 0
\end{array}\right)\left(\begin{array}{c}
t(s) \\
n(s) \\
b_{1}(s) \\
b_{2}(s)
\end{array}\right)
\end{aligned}
$$


where $\left(\cdot=\frac{d}{d s}\right),\left\{t, n, b_{1}, b_{2}\right\}, k_{1}, k_{2}$ and $k_{3}$ are the moving Frenet frame and the natural curvature functions respectively. Additionally, the vectors $t, n, b_{1}$ and $b_{2}$ satisfying the equations $\quad \mathfrak{F}(t, t)=\mathfrak{F}(n, n)=\mathfrak{F}\left(b_{1}, b_{1}\right)=$ $-\mathfrak{F}\left(b_{2}, b_{2}\right)=1$, and $\quad \mathfrak{F}(t, n)=\mathfrak{F}\left(t, b_{1}\right)=$ $\mathfrak{F}\left(t, b_{2}\right)=\mathfrak{F}\left(n, b_{1}\right)=\mathfrak{F}\left(n, b_{2}\right)=\mathfrak{F}\left(b_{1}, b_{2}\right)=$ 0 .

For any arbitrary spacelike curve $\zeta=\zeta(s)$ in 4-dimensional Minkowski space $\mathbb{R}_{1}^{4}$ satisfying Eq. (1), the Frenet apparatus of $\zeta$ can be formed as follows [17]:

$$
\begin{gathered}
t=\frac{\dot{\zeta}}{\|\dot{\zeta}\|}, \\
n=\frac{\|\dot{\zeta}\|^{2} \cdot \ddot{\zeta}-\mathfrak{F}(\dot{\zeta}, \ddot{\zeta}) \cdot \dot{\zeta}}{\|\| \dot{\zeta}\left\|^{2} \cdot \ddot{\zeta}-\mathfrak{F}(\dot{\zeta}, \ddot{\zeta}) \cdot \dot{\zeta}\right\|}, \\
b_{1}=\mu n \wedge t \wedge b_{2}, \\
k_{1}=\frac{\|\| \dot{\|}\left\|^{2} \cdot \ddot{\zeta}-\mathfrak{F}(\dot{\zeta}, \ddot{\zeta}) \cdot \dot{\zeta}\right\|}{\|t \wedge n \wedge \ddot{\zeta}\|^{4}}, \\
k_{2}=\frac{\|t \wedge n \wedge \ddot{\zeta}\| \cdot\|\dot{\zeta}\|}{\|\| \dot{\zeta}\left\|^{2} \cdot \ddot{\zeta}-\mathfrak{F}(\dot{\zeta}, \ddot{\zeta}) \cdot \dot{\zeta}\right\|}, \\
k_{3}=\frac{\tilde{F}\left(\zeta^{(4)}, b_{2}\right)}{\|t \wedge n \wedge \ddot{\zeta}\| \cdot\|\dot{\zeta}\|},
\end{gathered}
$$

where $\mu$ is taken \pm 1 to make the determinant of matrix $\left\{t, n, b_{1}, b_{2}\right\}$ equal \pm 1 .

Let be a regular spacelike curve lying fully on a spacelike surface $\Omega$ in Minkowski 4-space $\mathbb{R}_{1}^{4}$. We define the equiform parameter of $\zeta(s)$ by $\sigma=\int k_{1} d s$, where $\rho=\frac{1}{k_{1}}$ is the radius of curvature ofthe curve $\zeta$. Then, we have $\rho=\frac{d s}{d \sigma}$ Let $\mathcal{D}$ be a homothetic with the center in the origin and the coefficient $\lambda$. If we put $\zeta^{*}=$ $\mathcal{D}(\zeta)$, then it follows

$$
s^{*}=\lambda s \text { and } \rho^{*}=\lambda \rho \text {, }
$$

where $s^{*}$ is the arc-length parameter of $\zeta^{*}$ and $\rho^{*}$ is the radius of curvature of this curve.
Hence, $\sigma$ is an equiform invariant parameter of $\zeta[6]$. We recall $\left[T, \eta, \xi_{1}, \xi_{2}\right]$ be the moving equiform Frenet frame of the curve $\zeta$ where $T(\sigma)=\rho t(s)$ is the equiform tangent vector, $\eta(\sigma)=\rho n(s)$ is the equiform principal normal vector $\xi_{1}(\sigma)=\rho b_{1}(s) r$, is the equiform first binormal vector and $\xi_{2}(\sigma)=\rho b_{2}(s)$ is the equiform second binormal vector. Additionally, the equiform curvatures of the curve $\zeta=\zeta(\sigma)$ are defined by $k_{1}(\sigma)=$ $\dot{\rho}, k_{2}(\sigma)=\left(\frac{k_{2}}{k_{1}}\right)$ and $k_{3}(\sigma)=\left(\frac{k_{3}}{k_{1}}\right)$. Thus, the equiform Frenet formulas in $\mathbb{R}_{1}^{4}$ have the following frame [2]:

$$
\begin{aligned}
& \left(\begin{array}{l}
T^{\prime}(\sigma) \\
\eta^{\prime}(\sigma) \\
\xi_{1}^{\prime}(\sigma) \\
\xi_{2}^{\prime}(\sigma)
\end{array}\right) \\
& =\left(\begin{array}{cccl}
k_{1}(\sigma) & 1 & 0 & 0 \\
-1 & k_{1}(\sigma) & k_{2}(\sigma) & 0 \\
0 & -k_{2}(\sigma) & k_{1}(\sigma) & k_{3}(\sigma) \\
0 & 0 & k_{3}(\sigma) & k_{1}(\sigma)
\end{array}\right)\left(\begin{array}{l}
T(\sigma) \\
\eta(\sigma) \\
\xi_{1}(\sigma) \\
\xi_{2}(\sigma)
\end{array}\right)
\end{aligned}
$$

where $\left({ }^{\prime}=\frac{d}{d \sigma}\right), \mathfrak{F}(T, T)=\mathfrak{F}(\eta, \eta)=$ $\mathfrak{F}\left(\xi_{1}, \xi_{1}\right)=-\mathfrak{F}\left(\xi_{2}, \xi_{2}\right)=\rho^{2}$ and $\mathfrak{F}(T, \eta)=$ $\mathfrak{F}\left(T, \xi_{1}\right)=\mathfrak{F}\left(T, \xi_{2}\right)=\mathfrak{F}\left(\eta, \xi_{1}\right)=\mathfrak{F}\left(\eta, \xi_{2}\right)=0$.

\section{Main Results}

Through out this paper, we assume that the one parameter family of spacelike curves on a spacelike surface $\Omega$ in $\mathbb{R}_{1}^{4}$ is

$$
\zeta:[0, l] \times[0, w] \rightarrow \Omega \subset \mathbb{R}_{1}^{4},
$$

where $l$ is the arc-length of initial curve. Let $u$ be the curve parametrization variable $0<u<$ $l$. Then, the arc-length of $\zeta$ is given by

$$
s(u)=\int_{0}^{u}\left\|\frac{\partial \zeta}{\partial u}\right\| d u,
$$

where $\left\|\frac{\partial \zeta}{\partial u}\right\|=\| \mathcal{D}\left(\frac{\partial \zeta}{\partial u}, \frac{\partial \zeta}{\partial u} \|^{\frac{1}{2}}\right.$. The operator $\frac{\partial}{\partial s}$ is given in terms of $u$ by

$$
\frac{\partial}{\partial s}=\frac{1}{v} \frac{\partial}{\partial u}
$$

where $v=\left\|\frac{\partial \zeta}{\partial u}\right\|$. the arc-length parameter is $d s=v d u$. We can be represented any flows of spacelike curve $\zeta$ as

$$
\frac{\partial \zeta}{\partial t}=\mathfrak{R}_{1} T+\mathfrak{R}_{2} \eta+\mathfrak{R}_{3} \xi_{1}+\Re_{4} \xi_{2},
$$


Where $\mathfrak{R}_{1}, \mathfrak{R}_{2}, \mathfrak{R}_{3}$ and $\mathfrak{R}_{4}$ are smooth functions of arc-length and time $[5,9,12,13,16]$.

Let the arc-length variation be

$$
s(u, t)=\int_{0}^{u} v d u \text {, }
$$

In 4-dimensional Minkowski space $\mathbb{R}_{1}^{4}$, the requirement that the spacelike curve be not subject to any elongation or compression by the condition

$$
\frac{\partial}{\partial t} s(u, t)=\int_{0}^{u} \frac{\partial v}{\partial t} d u=0
$$

Definition 3.1. A curve evolution $\zeta(u . t)$ and its flow $\frac{\partial \zeta}{\partial t}$ on the spacelike surface $\Omega$ in $\mathbb{R}_{1}^{4}$ are said to be inextensible if

$$
\frac{\partial}{\partial t}\left\|\frac{\partial \zeta}{\partial u}\right\|=0
$$

Theorem 3.1. Let $\frac{\partial \zeta}{\partial t}=\mathfrak{R}_{1} T+\mathfrak{R}_{2} \eta+\mathfrak{R}_{3} \xi_{1}+$ $\mathfrak{R}_{4} \xi_{2}$, be a smooth inextensible flow of the spacelike curve $\zeta$ according to equiform frame in 4-dimensional Minkowski space $\mathbb{R}_{1}^{4}$. The flow is inextensible if and only if

$$
\frac{\partial \Re_{1}}{\partial u}=v\left(\Re_{2}-\Re_{1} k_{1}\right),
$$

where $\mathfrak{R}_{1}, \mathfrak{R}_{2}, \mathfrak{R}_{3}$ and $\mathfrak{R}_{4}$ are smooth functions of arc-length and time.

Proof. Assume that $\frac{\partial \zeta}{\partial t}$ is inextensible flow in $\mathbb{R}_{1}^{4}$. Then

$\frac{\partial}{\partial t} s(u, t)=\int_{0}^{u} \frac{\partial v}{\partial t} d u$

$$
=\int_{0}^{u}\left(\frac{\partial \Re_{1}}{\partial u}+\Re_{1} v k_{1}-\Re_{2} v\right) d u
$$$$
=0 \text {. }
$$

Substituting Eq. (7) in Eq. (9), we have Eq. (8) holds, this complete the proof.

Now, we restrict ourselves to arc-length parametrized curves. That is $v=1$ and the local coordinate $\mathrm{s}$ corresponding to the curve arc-length.

Theorem 3.2.Let $\frac{\partial \zeta}{\partial t}$ be a smooth inextensible flow of the spacelike curve $\zeta$ according to equiform frame in 4-dimensional Minkowski space $\mathbb{R}_{1}^{4}$. We have

$$
\begin{aligned}
\frac{\partial T}{\partial t}=\left(\frac{\partial \Re_{2}}{\partial s}+\right. & \left.\Re_{1}+\Re_{2} k_{1}-\Re_{3} k_{2}\right) \eta \\
& +\left(\frac{\partial \Re_{3}}{\partial s}+\Re_{2} k_{2}+\Re_{3} k_{1}\right. \\
& \left.+\mathfrak{R}_{4} k_{3}\right) \xi_{1} \\
& +\left(\frac{\partial \Re_{4}}{\partial s}+\Re_{3} k_{3}\right. \\
& \left.+\mathfrak{R}_{4} k_{1}\right) \xi_{2},
\end{aligned}
$$

where $\mathfrak{R}_{1}, \mathfrak{R}_{2}, \mathfrak{R}_{3}$ and $\mathfrak{R}_{4}$ are smooth functions of arc-length and time.

Proof. Using the definition of $\zeta$, we have

$$
\begin{aligned}
& \frac{\partial T}{\partial t} \\
& =\left(\frac{\partial \mathfrak{R}_{1}}{\partial s}+\mathfrak{R}_{1} k_{1}-\mathfrak{R}_{2}\right) T \\
& +\left(\frac{\partial \mathfrak{R}_{2}}{\partial s}+\mathfrak{R}_{1}+\mathfrak{R}_{2} k_{1}-\mathfrak{R}_{3} k_{2}\right) \eta \\
& +\left(\frac{\partial \mathfrak{R}_{3}}{\partial s}+\mathfrak{R}_{2} k_{2}+\mathfrak{R}_{3} k_{1}+\mathfrak{R}_{4} k_{3}\right) \xi_{1} \\
& +\left(\frac{\partial \mathfrak{R}_{4}}{\partial s}+\mathfrak{R}_{3} k_{3}\right. \\
& \left.+\mathfrak{R}_{4} k_{1}\right) \xi_{2},
\end{aligned}
$$

Substituting Eq. (8) in Eq. (10), then the Eq. (11) holds, which complete the proof.

Lemma 3.1. Let $\frac{\partial \zeta}{\partial t}$ be a smooth inextensible flow of the spacelike curve $\zeta$ according to equiform frame in 4-dimensional Minkowski space $\mathbb{R}_{1}^{4}$, then the evolution of $k_{1}$ satisfy the partial differential equation

$$
\frac{\partial k_{1}}{\partial t}=0 \text {. }
$$

Proof. Assume that $\frac{\partial \zeta}{\partial t}$ is inextensible flow in $\mathbb{R}_{1}^{4}$. Thus, we have 


$$
\begin{aligned}
& \frac{\partial}{\partial s} \frac{\partial T}{\partial t}=-\left[\frac{\partial \Re_{2}}{\partial s}+\Re_{1}+\mathfrak{R}_{2} k_{1}-\mathfrak{R}_{3} k_{2}\right] T \\
& +\left[\frac { \partial } { \partial s } \left(\frac{\partial \Re_{2}}{\partial s}+\Re_{1}+\Re_{2} k_{1}\right.\right. \\
& \left.-\mathfrak{R}_{3} k_{2}\right) \\
& +k_{1}\left(\frac{\partial \mathfrak{R}_{2}}{\partial s}+\mathfrak{R}_{1}+\mathfrak{R}_{2} k_{1}\right. \\
& \left.-\Re_{3} k_{2}\right) \\
& -k_{2}\left(\frac{\partial \mathfrak{R}_{3}}{\partial s}+\mathfrak{R}_{2} k_{2}+\mathfrak{R}_{3} k_{1}\right. \\
& \left.\left.+\mathfrak{R}_{4} k_{3}\right)\right] \eta \\
& +\left[\frac { \partial } { \partial s } \left(\frac{\partial \mathfrak{R}_{3}}{\partial s}+\mathfrak{R}_{2} k_{2}+\mathfrak{R}_{3} k_{1}\right.\right. \\
& \left.+\mathfrak{R}_{4} k_{3}\right) \\
& +k_{2}\left(\frac{\partial \Re_{2}}{\partial s}+\mathfrak{R}_{1}+\mathfrak{R}_{2} k_{1}\right. \\
& \left.-\Re_{3} k_{2}\right) \\
& +k_{1}\left(\frac{\partial \mathfrak{R}_{3}}{\partial s}+\mathfrak{R}_{2} k_{2}+\mathfrak{R}_{3} k_{1}\right. \\
& \left.+\mathfrak{R}_{4} k_{3}\right) \\
& \left.+k_{3}\left(\frac{\partial \Re_{4}}{\partial s}+\Re_{3} k_{3}+\Re_{4} k_{1}\right)\right] \xi_{1} \\
& +\left[\frac{\partial}{\partial s}\left(\frac{\partial \mathfrak{R}_{4}}{\partial s}+\mathfrak{R}_{3} k_{3}+\mathfrak{R}_{4} k_{1}\right)\right. \\
& +k_{3}\left(\frac{\partial \Re_{3}}{\partial s}+\mathfrak{R}_{2} k_{2}+\mathfrak{R}_{3} k_{1}\right. \\
& \left.+\mathfrak{R}_{4} k_{3}\right) \\
& +k_{1}\left(\frac{\partial \mathfrak{R}_{4}}{\partial s}+\mathfrak{R}_{3} k_{3}\right. \\
& \left.\left.+\mathfrak{R}_{4} k_{1}\right)\right] \xi_{2}
\end{aligned}
$$

On the other hand, From Eq. (2) we have

$$
\begin{aligned}
& \frac{\partial}{\partial t} \frac{\partial T}{\partial s}=\frac{\partial}{\partial t}\left(k_{1} T+\eta\right) \\
& =\frac{\partial k_{1}}{\partial t} T+k_{1} \frac{\partial T}{\partial t} \\
& +\frac{\partial \eta}{\partial t} .
\end{aligned}
$$

From Eqs. (13) and (14), we see that $-7=0$. This complete the proof.

Theorem 3.3. Let $\frac{\partial \zeta}{\partial t}$ be a smooth inextensible flow of the spacelike curve $\zeta$ according to equiform frame in 4-dimensional Minkowski space $\mathbb{R}_{1}^{4}$. We have

$$
\begin{aligned}
& \frac{\partial \eta}{\partial t}=\left[\frac{\partial \Re_{2}}{\partial s}+\Re_{1}+\mathfrak{R}_{2} k_{1}-\Re_{3} k_{2}\right] T \\
& +\left[\frac { \partial } { \partial t } \left(\frac{\partial \Re_{3}}{\partial s}+\Re_{2} k_{2}+\Re_{3} k_{1}\right.\right. \\
& \left.+\mathfrak{R}_{4} k_{3}\right) \\
& +k_{2}\left(\frac{\partial \mathfrak{R}_{2}}{\partial s}+\mathfrak{R}_{1}+\mathfrak{R}_{2} k_{1}\right. \\
& \left.-\mathfrak{R}_{3} k_{2}\right) \\
& \left.+k_{3}\left(\frac{\partial \Re_{4}}{\partial s}+\Re_{3} k_{3}+\Re_{4} k_{1}\right)\right] \xi_{1} \\
& +\left[\frac{\partial}{\partial t}\left(\frac{\partial \mathfrak{R}_{4}}{\partial s}+\mathfrak{R}_{3} k_{3}+\mathfrak{R}_{4} k_{1}\right)\right. \\
& +k_{3}\left(\frac{\partial \mathfrak{R}_{3}}{\partial s}+\mathfrak{R}_{2} k_{2}+\mathfrak{R}_{3} k_{1}\right. \\
& \left.\left.+\mathfrak{R}_{4} k_{3}\right)\right] \xi_{2} \text {, }
\end{aligned}
$$

where $\mathfrak{R}_{1}, \mathfrak{R}_{2}, \mathfrak{R}_{3}$ and $\mathfrak{R}_{4}$ are smooth functions of arc-length and time.

Proof. Let $\frac{\partial \zeta}{\partial t}$ is inextensible flow of a spacelike curve $\zeta$ in $\mathbb{R}_{1}^{4}$. From Eq. (14), we have

$$
\begin{aligned}
& \frac{\partial \eta}{\partial t} \\
& =\frac{\partial}{\partial t} \frac{\partial T}{\partial s}-k_{1} \frac{\partial T}{\partial t} \\
& -\frac{\partial k_{1}}{\partial t} T .
\end{aligned}
$$

Substituting Eqs. (10), (12) and (13) in Eq. (16), we get

$$
\begin{aligned}
\frac{\partial \eta}{\partial t}=-\left[\frac{\partial \Re_{2}}{\partial s}\right. & \left.+\Re_{1}+\mathfrak{R}_{2} k_{1}-\mathfrak{R}_{3} k_{2}\right] T \\
& +\left[\frac { \partial } { \partial s } \left(\frac{\partial \mathfrak{R}_{2}}{\partial s}+\mathfrak{R}_{1}+\mathfrak{R}_{2} k_{1}\right.\right. \\
& \left.-\mathfrak{R}_{3} k_{2}\right) \\
& -k_{2}\left(\frac{\partial \mathfrak{R}_{3}}{\partial s}+\mathfrak{R}_{2} k_{2}+\mathfrak{R}_{3} k_{1}\right. \\
& \left.\left.+\mathfrak{R}_{4} k_{3}\right)\right] \eta
\end{aligned}
$$




$$
\begin{aligned}
+\left[\frac{\partial}{\partial s}\left(\frac{\partial \Re_{3}}{\partial s}+\mathfrak{R}_{2} k_{2}+\mathfrak{R}_{3} k_{1}+\mathfrak{R}_{4} k_{3}\right)\right. \\
+k_{2}\left(\frac{\partial \Re_{2}}{\partial s}+\mathfrak{R}_{1}+\mathfrak{R}_{2} k_{1}\right. \\
\left.\quad-\mathfrak{R}_{3} k_{2}\right) \\
\left.+k_{3}\left(\frac{\partial \mathfrak{R}_{4}}{\partial s}+\mathfrak{R}_{3} k_{3}+\mathfrak{R}_{4} k_{1}\right)\right] \xi_{1} \\
+\left[\frac{\partial}{\partial s}\left(\frac{\partial \Re_{4}}{\partial s}+\mathfrak{R}_{3} k_{3}+\mathfrak{R}_{4} k_{1}\right)\right. \\
+k_{3}\left(\frac{\partial \mathfrak{R}_{3}}{\partial s}+\mathfrak{R}_{2} k_{2}+\mathfrak{R}_{3} k_{1}\right. \\
\left.\left.+\mathfrak{R}_{4} k_{3}\right)\right] \xi_{2}
\end{aligned}
$$

Then, one can easily say that

$$
\mathfrak{F}\left(\eta, \frac{\partial \eta}{\partial t}\right)=0 \text {. }
$$

Asa consequence of the above equation

$$
\begin{aligned}
\frac{\partial \eta}{\partial t}=-\left[\frac{\partial \mathfrak{R}_{2}}{\partial s}\right. & \left.+\mathfrak{R}_{1}+\mathfrak{R}_{2} k_{1}-\mathfrak{R}_{3} k_{2}\right] T \\
& +\left[\frac { \partial } { \partial s } \left(\frac{\partial \mathfrak{R}_{3}}{\partial s}+\mathfrak{R}_{2} k_{2}+\mathfrak{R}_{3} k_{1}\right.\right. \\
& \left.+\mathfrak{R}_{4} k_{3}\right) \\
& +k_{2}\left(\frac{\partial \mathfrak{R}_{2}}{\partial s}+\mathfrak{R}_{1}+\mathfrak{R}_{2} k_{1}\right. \\
& \left.-\mathfrak{R}_{3} k_{2}\right) \\
& \left.+k_{3}\left(\frac{\partial \mathfrak{R}_{4}}{\partial s}+\mathfrak{R}_{3} k_{3}+\mathfrak{R}_{4} k_{1}\right)\right] \xi_{1} \\
& +\left[\frac{\partial}{\partial s}\left(\frac{\partial \mathfrak{R}_{4}}{\partial s}+\mathfrak{R}_{3} k_{3}+\mathfrak{R}_{4} k_{1}\right)\right. \\
& +k_{3}\left(\frac{\partial \mathfrak{R}_{3}}{\partial s}+\mathfrak{R}_{2} k_{2}+\mathfrak{R}_{3} k_{1}\right. \\
& \left.\left.+\mathfrak{R}_{4} k_{3}\right)\right] \xi_{2}
\end{aligned}
$$

Theorem 3.4. Let $\frac{\partial \zeta}{\partial t}$ be a smooth inextensible flow of the spacelike curve $\zeta$ according to equiform frame in 4-dimensional Minkowski space $\mathbb{R}_{1}^{4}$. Then

$$
\begin{aligned}
& \frac{\partial \xi_{1}}{\partial t}=\frac{-2}{k_{2}}\left[\frac{\partial}{\partial s}\left(\frac{\partial \Re_{2}}{\partial s}+\Re_{1}+\Re_{2} k_{1}-\Re_{3} k_{2}\right)\right] T \\
& +\frac{1}{k_{2}}\left[2 \left(\frac{\partial \Re_{2}}{\partial s}+\Re_{1}+\Re_{2} k_{1}\right.\right. \\
& \left.-\mathfrak{R}_{3} k_{2}\right) \\
& +\frac{\partial}{\partial s}\left[\frac { \partial } { \partial s } \left(\frac{\partial \Re_{2}}{\partial s}+\Re_{1}+\Re_{2} k_{1}\right.\right. \\
& \left.-\mathfrak{R}_{3} k_{2}\right) \\
& -k_{2}\left(\frac{\partial \mathfrak{R}_{3}}{\partial s}+\mathfrak{R}_{2} k_{2}+\mathfrak{R}_{3} k_{1}\right. \\
& \left.\left.+\mathfrak{R}_{4} k_{3}\right)\right] \\
& -k_{2}\left[\frac { \partial } { \partial s } \left(\frac{\partial \Re_{3}}{\partial s}+\mathfrak{R}_{2} k_{2}+\mathfrak{R}_{3} k_{1}\right.\right. \\
& \left.+\mathfrak{R}_{4} k_{3}\right) \\
& +k_{2}\left(\frac{\partial \Re_{2}}{\partial s}+\mathfrak{R}_{1}+\mathfrak{R}_{2} k_{1}\right. \\
& \left.-\Re_{3} k_{2}\right) \\
& \left.\left.+k_{3}\left(\frac{\partial \Re_{4}}{\partial s}+\Re_{3} k_{3}+\Re_{4} k_{1}\right)\right]\right] \eta \\
& +\frac{1}{k_{2}}\left[k _ { 3 } \left[\frac { \partial } { \partial s } \left(\frac{\partial \Re_{3}}{\partial s}+\mathfrak{R}_{2} k_{2}\right.\right.\right. \\
& \left.+\mathfrak{R}_{3} k_{1}+\mathfrak{R}_{4} k_{3}\right) \\
& +k_{2}\left(\frac{\partial \Re_{2}}{\partial s}+\Re_{1}+\mathfrak{R}_{2} k_{1}\right. \\
& \left.-\mathfrak{R}_{3} k_{2}\right) \\
& \left.\left.+k_{3}\left(\frac{\partial \mathfrak{R}_{4}}{\partial s}+\mathfrak{R}_{3} k_{3}+\mathfrak{R}_{4} k_{1}\right)\right]\right]
\end{aligned}
$$




$$
\begin{aligned}
+\frac{\partial}{\partial s}\left[\frac { \partial } { \partial s } \left(\frac{\partial \Re_{4}}{\partial s}\right.\right. & \left.+\mathfrak{R}_{3} k_{3}+\mathfrak{R}_{4} k_{1}\right) \\
& +k_{3}\left(\frac{\partial \mathfrak{R}_{3}}{\partial s}+\mathfrak{R}_{2} k_{2}+\mathfrak{R}_{3} k_{1}\right. \\
& \left.\left.+\mathfrak{R}_{4} k_{3}\right)\right] \\
& -\left(\frac{\partial \mathfrak{R}_{4}}{\partial s}+\mathfrak{R}_{3} k_{3}\right. \\
& \left.+\mathfrak{R}_{4} k_{1}\right) \xi_{2}
\end{aligned}
$$

where $\mathfrak{R}_{1}, \mathfrak{R}_{2}, \mathfrak{R}_{3}$ and $\mathfrak{R}_{4}$ are smooth functions of arc-length and time.

Proof. Assume that $\frac{\partial \zeta}{\partial t}$ is inextensible flow of a spacelike curve $\zeta$ in $\mathbb{R}_{1}^{4}$. We have

$$
\begin{aligned}
& \frac{\partial}{\partial s} \frac{\partial \eta}{\partial t}=-\left[2 \frac{\partial}{\partial s}\left(\frac{\partial \Re_{2}}{\partial s}+\Re_{1}+\mathfrak{R}_{2} k_{1}-\mathfrak{R}_{3} k_{2}\right)\right. \\
& +k_{1}\left(\frac{\partial \mathfrak{R}_{2}}{\partial s}+\mathfrak{R}_{1}+\mathfrak{R}_{2} k_{1}\right. \\
& \left.\left.-\mathfrak{R}_{3}\right)\right] T \\
& +\left[\frac { \partial } { \partial s } \left[\frac { \partial } { \partial s } \left(\frac{\partial \Re_{2}}{\partial s}+\Re_{1}+\Re_{2} k_{1}\right.\right.\right. \\
& \left.-\Re_{3} k_{2}\right) \\
& -k_{2}\left(\frac{\partial \mathfrak{R}_{3}}{\partial s}+\mathfrak{R}_{2} k_{2}+\mathfrak{R}_{3} k_{1}\right. \\
& \left.\left.+\mathfrak{R}_{4} k_{3}\right)\right]+\frac{\partial \mathfrak{R}_{2}}{\partial s} \Re_{1}+\mathfrak{R}_{2} k_{1} \\
& -\Re_{3} k_{2} \\
& -k_{2}\left[\frac { \partial } { \partial s } \left(\frac{\partial \Re_{3}}{\partial s}+\Re_{2} k_{2}+\Re_{3} k_{1}\right.\right. \\
& \left.+\mathfrak{R}_{4} k_{3}\right) \\
& +k_{2}\left(\frac{\partial \Re_{2}}{\partial s}+\Re_{1}+\Re_{2} k_{1}-\Re_{3} k_{2}\right) \\
& \left.+k_{3}\left(\frac{\partial \Re_{4}}{\partial s}+\Re_{3} k_{3}+\mathfrak{R}_{4} k_{1}\right)\right] \\
& +k_{1}\left[\frac { \partial } { \partial s } \left(\frac{\partial \Re_{2}}{\partial s}+\Re_{1}+\Re_{2} k_{1}\right.\right. \\
& \left.-\mathfrak{R}_{3} k_{2}\right) \\
& -k_{2}\left(\frac{\partial \mathfrak{R}_{3}}{\partial s}+\mathfrak{R}_{2} k_{2}+\mathfrak{R}_{3} k_{1}\right. \\
& \left.\left.\left.+\Re_{4} k_{3}\right)\right]\right] \eta
\end{aligned}
$$

$+\left[k_{2}\left[\frac{\partial}{\partial s}\left(\frac{\partial \Re_{2}}{\partial s}+\Re_{1}+\Re_{2} k_{1}-\Re_{3} k_{2}\right)\right.\right.$

$-k_{2}\left(\frac{\partial \Re_{3}}{\partial s}+\mathfrak{R}_{2} k_{2}+\mathfrak{R}_{3} k_{1}\right.$

$\left.\left.+\Re_{4} k_{3}\right)\right]$

$+\frac{\partial}{\partial s}\left(\frac{\partial \Re_{3}}{\partial s}+\mathfrak{R}_{2} k_{2}+\mathfrak{R}_{3} k_{1}\right.$

$\left.+\mathfrak{R}_{4} k_{3}\right)$

$+k_{2}\left(\frac{\partial \mathfrak{R}_{2}}{\partial s}+\mathfrak{R}_{1}+\mathfrak{R}_{2} k_{1}\right.$

$\left.-\mathfrak{R}_{3} k_{2}\right)$

$+k_{3}\left(\frac{\partial \Re_{4}}{\partial s}+\mathfrak{R}_{3} k_{3}+\mathfrak{R}_{4} k_{1}\right)$

$+k_{1}\left[\frac{\partial}{\partial s}\left(\frac{\partial \Re_{3}}{\partial s}+\Re_{2} k_{2}+\Re_{3} k_{1}\right.\right.$

$\left.+\mathfrak{R}_{4} k_{3}\right)$

$+k_{2}\left(\frac{\partial \mathfrak{R}_{2}}{\partial s}+\mathfrak{R}_{1}+\mathfrak{R}_{2} k_{1}\right.$

$\left.-\Re_{3} k_{2}\right)$

$\left.+k_{3}\left(\frac{\partial \Re_{4}}{\partial s}+\mathfrak{R}_{3} k_{3}+\mathfrak{R}_{4} k_{1}\right)\right]$

$+k_{3}\left[\frac{\partial}{\partial s}\left(\frac{\partial \Re_{4}}{\partial s}+\Re_{3} k_{3}+\Re_{4} k_{1}\right)\right.$

$+k_{3}\left(\frac{\partial \mathfrak{R}_{3}}{\partial s}+\mathfrak{R}_{2} k_{2}+\mathfrak{R}_{3} k_{1}\right.$

$\left.\left.\left.+\mathfrak{R}_{4} k_{3}\right)\right]\right] \xi_{1}$ 


$$
\begin{aligned}
& +\left[k _ { 3 } \left[\frac{\partial}{\partial s}\left(\frac{\partial \Re_{3}}{\partial s}+\mathfrak{R}_{2} k_{2}+\mathfrak{R}_{3} k_{1}+\mathfrak{R}_{4} k_{3}\right)\right.\right. \\
& +k_{2}\left(\frac{\partial \Re_{2}}{\partial s}+\Re_{1}+\mathfrak{R}_{2} k_{1}-\mathfrak{R}_{3} k_{2}\right) \\
& \left.+k_{3}\left(\frac{\partial \mathfrak{R}_{4}}{\partial s}+\mathfrak{R}_{3} k_{3}+\mathfrak{R}_{4} k_{1}\right)\right] \\
& +\frac{\partial}{\partial s}\left[\frac{\partial}{\partial s}\left(\frac{\partial \Re_{4}}{\partial s}+\Re_{3} k_{3}+\Re_{4} k_{1}\right)\right. \\
& \left.+k_{3}\left(\frac{\partial \mathfrak{R}_{3}}{\partial s}+\mathfrak{R}_{2} k_{2}+\mathfrak{R}_{3} k_{1}+\mathfrak{R}_{4} k_{3}\right)\right] \\
& +k_{1}\left[\frac{\partial}{\partial s}\left(\frac{\partial \Re_{4}}{\partial s}+\Re_{3} k_{3}+\mathfrak{R}_{4} k_{1}\right)\right. \\
& +k_{3}\left(\frac{\partial \Re_{3}}{\partial s}+\Re_{2} k_{2}+\Re_{3} k_{1}\right. \\
& \left.\left.\left.+\Re_{4} k_{3}\right)\right]\right] \xi_{2} \text {. }
\end{aligned}
$$

Underthe assumption of spacelike curve $\zeta$, we have

$$
\begin{aligned}
\frac{\partial}{\partial s} \frac{\partial \eta}{\partial t}=-\left[k_{1}\right. & \left.\left(\frac{\partial \mathfrak{R}_{2}}{\partial s}+\mathfrak{R}_{1}+\mathfrak{R}_{2} k_{1}-\mathfrak{R}_{3} k_{2}\right)\right] T \\
& -\left[\frac{\partial \mathfrak{R}_{2}}{\partial s}+\mathfrak{R}_{1}+\mathfrak{R}_{2} k_{1}\right. \\
& \left.-\mathfrak{R}_{3} k_{2}\right] \eta \\
& +\left[k _ { 1 } \left[\frac { \partial } { \partial s } \left(\frac{\partial \mathfrak{R}_{2}}{\partial s}+\mathfrak{R}_{1}+\mathfrak{R}_{2} k_{1}\right.\right.\right. \\
& \left.-\mathfrak{R}_{3} k_{2}\right) \\
& +k_{2}\left(\frac{\partial \mathfrak{R}_{2}}{\partial s}+\mathfrak{R}_{1}+\mathfrak{R}_{2} k_{1}\right. \\
& \left.-\mathfrak{R}_{3} k_{2}\right) \\
& +k_{3}\left(\frac{\partial \mathfrak{R}_{4}}{\partial s}+\mathfrak{R}_{3} k_{3}+\mathfrak{R}_{4} k_{1}\right) \\
& -\left(\frac{\partial \mathfrak{R}_{3}}{\partial s}+\mathfrak{R}_{2} k_{2}+\mathfrak{R}_{3} k_{1}\right. \\
& \left.\left.\left.+\mathfrak{R}_{4} k_{3}\right)\right]-\frac{\partial k_{2}}{\partial t}\right] \xi_{1}
\end{aligned}
$$

$$
\begin{aligned}
+\left[k _ { 1 } \left[\frac { \partial } { \partial s } \left(\frac{\partial \Re_{4}}{\partial s}\right.\right.\right. & \left.+\Re_{3} k_{3}+\Re_{4} k_{1}\right) \\
& +k_{3}\left(\frac{\partial \Re_{3}}{\partial s}+\Re_{2} k_{2}+\Re_{3} k_{1}\right. \\
& \left.\left.+\mathfrak{R}_{4} k_{3}\right)\right] \\
& \left.-\left(\frac{\partial \mathfrak{R}_{4}}{\partial s}+\mathfrak{R}_{3} k_{3}+\mathfrak{R}_{4} k_{1}\right)\right] \xi_{2} \\
& +k_{2} \frac{\partial \xi_{2}}{\partial t} .
\end{aligned}
$$

Then, it is follow that

$$
\begin{aligned}
& k_{2} \frac{\partial \xi_{2}}{\partial t}=-2\left[\frac { \partial } { \partial s } \left(\frac{\partial \Re_{2}}{\partial s}+\Re_{1}+\Re_{2} k_{1}\right.\right. \\
& \left.\left.-\mathfrak{R}_{3} k_{2}\right)\right] T \\
& +\left[\frac { \partial } { \partial s } \left[\frac { \partial } { \partial s } \left(\frac{\partial \Re_{2}}{\partial s}+\Re_{1}+\mathfrak{R}_{2} k_{1}\right.\right.\right. \\
& \left.-\mathfrak{R}_{3} k_{2}\right) \\
& -k_{2}\left(\frac{\partial \mathfrak{R}_{3}}{\partial s}+\mathfrak{R}_{2} k_{2}+\mathfrak{R}_{3} k_{1}\right. \\
& \left.\left.+\Re_{4} k_{3}\right)\right] \\
& +2\left(\frac{\partial \Re_{2}}{\partial s}+\Re_{1}+\Re_{2} k_{1}\right. \\
& \left.-\mathfrak{R}_{3} k_{2}\right) \\
& -k_{2}\left[\frac { \partial } { \partial s } \left(\frac{\partial \mathfrak{R}_{3}}{\partial s}+\mathfrak{R}_{2} k_{2}+\mathfrak{R}_{3} k_{1}\right.\right. \\
& \left.+\mathfrak{R}_{4} k_{3}\right) \\
& +k_{2}\left(\frac{\partial \mathfrak{R}_{2}}{\partial s}+\mathfrak{R}_{1}+\mathfrak{R}_{2} k_{1}\right. \\
& \left.-\mathfrak{R}_{3} k_{2}\right) \\
& \left.\left.+k_{3}\left(\frac{\partial \Re_{4}}{\partial s}+\Re_{3} k_{3}+\Re_{4} k_{1}\right)\right]\right] \eta
\end{aligned}
$$




$$
\begin{aligned}
& +\left[k_{2} \frac{\partial}{\partial s}\left(\frac{\partial \Re_{2}}{\partial s}+\Re_{1}+\Re_{2} k_{1}-\Re_{3} k_{2}\right)\right. \\
& -\left(k_{2}^{2}-1\right)\left(\frac{\partial \Re_{3}}{\partial s}+\mathfrak{R}_{2} k_{2}\right. \\
& \left.+\mathfrak{R}_{3} k_{1}+\mathfrak{R}_{4} k_{3}\right) \\
& +\frac{\partial}{\partial s}\left[\frac { \partial } { \partial s } \left(\frac{\partial \mathfrak{R}_{3}}{\partial s}+\mathfrak{R}_{2} k_{2}+\mathfrak{R}_{3} k_{1}\right.\right. \\
& \left.+\mathfrak{R}_{4} k_{3}\right) \\
& +k_{2}\left(\frac{\partial \mathfrak{R}_{2}}{\partial s}+\mathfrak{R}_{1}+\mathfrak{R}_{2} k_{1}\right. \\
& \left.-\Re_{3} k_{2}\right) \\
& \left.+k_{3}\left(\frac{\partial \Re_{4}}{\partial s}+\mathfrak{R}_{3} k_{3}+\mathfrak{R}_{4} k_{1}\right)\right] \\
& +k_{1}\left[\frac { \partial } { \partial s } \left(\frac{\partial \Re_{3}}{\partial s}+\mathfrak{R}_{2} k_{2}+\mathfrak{R}_{3} k_{1}\right.\right. \\
& \left.+\Re_{4} k_{3}\right) \\
& +\frac{\partial}{\partial s}\left(\frac{\partial \Re_{2}}{\partial s}+\Re_{1}+\mathfrak{R}_{2} k_{1}\right. \\
& \left.\left.-\Re_{3} k_{2}\right)\right] \\
& +k_{3}\left[\frac{\partial}{\partial s}\left(\frac{\partial \Re_{4}}{\partial s}+\Re_{3} k_{3}+\mathfrak{R}_{4} k_{1}\right)\right. \\
& +k_{3}\left(\frac{\partial \Re_{3}}{\partial s}+\mathfrak{R}_{2} k_{2}+\mathfrak{R}_{3} k_{1}\right. \\
& \left.\left.\left.+\mathfrak{R}_{4} k_{3}\right)\right]-\frac{\partial k_{2}}{\partial t}\right] \xi_{1}
\end{aligned}
$$

$$
\begin{aligned}
+\left[k _ { 3 } \left[\frac{\partial}{\partial s}\right.\right. & \left(\frac{\partial \Re_{3}}{\partial s}+\Re_{2} k_{2}+\Re_{3} k_{1}+\Re_{4} k_{3}\right) \\
& +k_{2}\left(\frac{\partial \Re_{2}}{\partial s}+\Re_{1}+\Re_{2} k_{1}\right. \\
& \left.-\Re_{3} k_{2}\right) \\
& \left.+k_{3}\left(\frac{\partial \Re_{4}}{\partial s}+\Re_{3} k_{3}+\Re_{4} k_{1}\right)\right] \\
& +\frac{\partial}{\partial s}\left[\frac{\partial}{\partial s}\left(\frac{\partial \Re_{4}}{\partial s}+\Re_{3} k_{3}+\Re_{4} k_{1}\right)\right. \\
& +k_{3}\left(\frac{\partial \Re_{3}}{\partial s}+\Re_{2} k_{2}+\Re_{3} k_{1}\right. \\
& \left.\left.+\Re_{4} k_{3}\right)\right] \\
& -\left(\frac{\partial \Re_{4}}{\partial s}+\Re_{3} k_{3}\right. \\
& \left.\left.+\Re_{4} k_{1}\right)\right] \xi_{2} .
\end{aligned}
$$

From the definition of flow

$$
\mathfrak{F}\left(\xi_{1}, \frac{\partial \xi_{1}}{\partial t}\right)=0 .
$$

Thus the proof of theorem is complete.

Lemma 3.2. Let $\frac{\partial \zeta}{\partial t}$ be a smooth inextensible flow of the spacelike curve $\zeta$ according to equiform frame in 4-dimensional Minkowski space $\mathbb{R}_{1}^{4}$. The evolution of $k_{2}$ satisfy the partial differential equation 


$$
\begin{aligned}
& \frac{\partial k_{2}}{\partial t} \\
& =k_{2} \frac{\partial}{\partial s}\left(\frac{\partial \mathfrak{R}_{2}}{\partial s}+\mathfrak{R}_{1}+\mathfrak{R}_{2} k_{1}-\mathfrak{R}_{3} k_{2}\right) \\
& -\left(k_{2}^{2}-1\right)\left(\frac{\partial \mathfrak{R}_{3}}{\partial s}+\mathfrak{R}_{2} k_{2}+\mathfrak{R}_{3} k_{1}+\mathfrak{R}_{4} k_{3}\right) \\
& +\frac{\partial}{\partial s}\left[\frac{\partial}{\partial s}\left(\frac{\partial \mathfrak{R}_{3}}{\partial s}+\mathfrak{R}_{2} k_{2}+\mathfrak{R}_{3} k_{1}+\mathfrak{R}_{4} k_{3}\right)\right. \\
& +k_{2}\left(\frac{\partial \mathfrak{R}_{2}}{\partial s}+\mathfrak{R}_{1}+\mathfrak{R}_{2} k_{1}-\mathfrak{R}_{3} k_{2}\right) \\
& \left.+k_{3}\left(\frac{\partial \mathfrak{R}_{4}}{\partial s}+\mathfrak{R}_{3} k_{3}+\mathfrak{R}_{4} k_{1}\right)\right] \\
& +k_{1}\left[\frac{\partial}{\partial s}\left(\frac{\partial \mathfrak{R}_{3}}{\partial s}+\mathfrak{R}_{2} k_{2}+\mathfrak{R}_{3} k_{1}+\mathfrak{R}_{4} k_{3}\right)\right. \\
& \left.+\frac{\partial}{\partial s}\left(\frac{\partial \mathfrak{R}_{2}}{\partial s}+\mathfrak{R}_{1}+\mathfrak{R}_{2} k_{1}-\mathfrak{R}_{3} k_{2}\right)\right] \\
& +k_{3}\left[\frac{\partial}{\partial s}\left(\frac{\partial \mathfrak{R}_{4}}{\partial s}+\mathfrak{R}_{3} k_{3}+\mathfrak{R}_{4} k_{1}\right)\right. \\
& +k_{3}\left(\frac{\partial \mathfrak{R}_{3}}{\partial s}+\mathfrak{R}_{2} k_{2}+\mathfrak{R}_{3} k_{1}\right. \\
& \left.\left.+\mathfrak{R}_{4} k_{3}\right)\right]
\end{aligned}
$$

Proof. It is obvious directly from Theorem(3.4).

This complete the proof.

Theorem 3.5. let $\frac{\partial \zeta}{\partial t}$ be a smooth inextensible flow of the spacelike curve $\zeta$ according to equiform frame in 4-dimensional Minkowski space $\mathbb{R}_{1}^{4}$. Then

$$
\begin{aligned}
& \frac{\partial \xi_{2}}{\partial t}=\frac{1}{k_{3}}\left[\frac{\partial f}{\partial s}-k_{2}\left(\frac{\partial \Re_{2}}{\partial s}+\Re_{1}+\mathfrak{R}_{2} k_{1}\right.\right. \\
& \left.\left.-\Re_{3} k_{2}\right)-g\right] T \\
& +\frac{1}{k_{3}}\left[\frac{\partial g}{\partial s}\right. \\
& -\left(k_{2}+\frac{2}{k_{2}}\right) \frac{\partial}{\partial s}\left(\frac{\partial \Re_{2}}{\partial s}+\Re_{1}\right. \\
& \left.+\mathfrak{R}_{2} k_{1}-\mathfrak{R}_{3} k_{2}\right) \\
& +\left(k_{2}^{2}-1\right)\left(\frac{\partial \Re_{3}}{\partial s}+\Re_{2} k_{2}\right. \\
& \left.+\mathfrak{R}_{3} k_{1}+\mathfrak{R}_{4} k_{3}\right) \\
& -\frac{\partial}{\partial s}\left[\frac { \partial } { \partial s } \left(\frac{\partial \Re_{3}}{\partial s}+\mathfrak{R}_{2} k_{2}+\mathfrak{R}_{3} k_{1}\right.\right. \\
& \left.+\mathfrak{R}_{4} k_{3}\right) \\
& +k_{2}\left(\frac{\partial \mathfrak{R}_{2}}{\partial s}+\mathfrak{R}_{1}+\mathfrak{R}_{2} k_{1}\right. \\
& \left.-\mathfrak{R}_{3} k_{2}\right) \\
& \left.+k_{3}\left(\frac{\partial \mathfrak{R}_{4}}{\partial s}+\mathfrak{R}_{3} k_{3}+\mathfrak{R}_{4} k_{1}\right)\right] \\
& -k_{1}\left[\frac { \partial } { \partial s } \left(\frac{\partial \Re_{3}}{\partial s}+\Re_{2} k_{2}+\Re_{3} k_{1}\right.\right. \\
& \left.+\mathfrak{R}_{4} k_{3}\right) \\
& +\frac{\partial}{\partial s}\left(\frac{\partial \Re_{2}}{\partial s}+\Re_{1}+\Re_{2} k_{1}\right. \\
& \left.\left.-\mathfrak{R}_{3} k_{2}\right)\right] \\
& -k_{3}\left[\frac{\partial}{\partial s}\left(\frac{\partial \Re_{4}}{\partial s}+\Re_{3} k_{3}+\Re_{4} k_{1}\right)\right. \\
& +k_{3}\left(\frac{\partial \Re_{3}}{\partial s}+\mathfrak{R}_{2} k_{2}+\mathfrak{R}_{3} k_{1}\right. \\
& \left.\left.\left.+\mathfrak{R}_{4} k_{3}\right)\right]\right] \eta
\end{aligned}
$$




$$
\begin{aligned}
& +\frac{1}{k_{3}}\left[h k_{3}\right. \\
& +k_{2}\left[\frac{\partial}{\partial s}\left(\frac{\partial \Re_{2}}{\partial s}+\Re_{1}+\Re_{2} k_{1}-\Re_{3} k_{2}\right)\right. \\
& +k_{1}\left(\frac{\partial \Re_{2}}{\partial s}+\Re_{1}+\Re_{2} k_{1}-\Re_{3} k_{2}\right) \\
& +k_{3} \frac{\partial}{\partial s}\left(\frac{\partial \Re_{4}}{\partial s}+\Re_{3} k_{3}+\Re_{4} k_{1}\right) \\
& +g]] \xi_{1} .
\end{aligned}
$$

Where

$$
\begin{aligned}
& f=-\frac{2}{k_{2}}\left[\frac{\partial}{\partial s}\left(\frac{\partial \Re_{2}}{\partial s}+\Re_{1}+\Re_{2} k_{1}-\mathfrak{R}_{3} k_{2}\right)\right] \text {, } \\
& g=\frac{1}{k_{2}}\left[2\left(\frac{\partial \Re_{2}}{\partial s}+\Re_{1}+\Re_{2} k_{1}-\Re_{3} k_{2}\right)\right. \\
& +\frac{\partial}{\partial s}\left[\frac { \partial } { \partial s } \left(\frac{\partial \Re_{2}}{\partial s}+\mathfrak{R}_{1}+\mathfrak{R}_{2} k_{1}\right.\right. \\
& \left.-\mathfrak{R}_{3} k_{2}\right) \\
& -k_{2}\left(\frac{\partial \Re_{3}}{\partial s}+\mathfrak{R}_{2} k_{2}+\mathfrak{R}_{3} k_{1}\right. \\
& \left.\left.+\mathfrak{R}_{4} k_{3}\right)\right] \\
& -k_{2}\left[\frac { \partial } { \partial s } \left(\frac{\partial \Re_{3}}{\partial s}+\mathfrak{R}_{2} k_{2}+\mathfrak{R}_{3} k_{1}\right.\right. \\
& \left.+\mathfrak{R}_{4} k_{3}\right) \\
& +k_{2}\left(\frac{\partial \Re_{2}}{\partial s}+\mathfrak{R}_{1}+\mathfrak{R}_{2} k_{1}\right. \\
& \left.-\mathfrak{R}_{3} k_{2}\right) \\
& \left.\left.+k_{3}\left(\frac{\partial \mathfrak{R}_{4}}{\partial s}+\mathfrak{R}_{3} k_{3}+\mathfrak{R}_{4} k_{1}\right)\right]\right] \text {, }
\end{aligned}
$$

$$
\begin{aligned}
h=\frac{1}{k_{2}}\left[k _ { 3 } \left[\frac{\partial}{\partial s}\right.\right. & \left(\frac{\partial \Re_{3}}{\partial s}+\mathfrak{R}_{2} k_{2}+\mathfrak{R}_{3} k_{1}+\mathfrak{R}_{4} k_{3}\right) \\
& +k_{2}\left(\frac{\partial \mathfrak{R}_{2}}{\partial s}+\mathfrak{R}_{1}+\mathfrak{R}_{2} k_{1}\right. \\
& \left.-\mathfrak{R}_{3} k_{2}\right) \\
& \left.+k_{3}\left(\frac{\partial \mathfrak{R}_{4}}{\partial s}+\mathfrak{R}_{3} k_{3}+\mathfrak{R}_{4} k_{1}\right)\right] \\
& +\frac{\partial}{\partial s}\left[\frac{\partial}{\partial s}\left(\frac{\partial \mathfrak{R}_{4}}{\partial s}+\mathfrak{R}_{3} k_{3}+\mathfrak{R}_{4} k_{1}\right)\right. \\
& +k_{3}\left(\frac{\partial \mathfrak{R}_{3}}{\partial s}+\mathfrak{R}_{2} k_{2}+\mathfrak{R}_{3} k_{1}\right. \\
& \left.\left.+\mathfrak{R}_{4} k_{3}\right)\right] \\
& \left.-\left(\frac{\partial \mathfrak{R}_{4}}{\partial s}+\mathfrak{R}_{3} k_{3}+\mathfrak{R}_{4} k_{1}\right)\right]
\end{aligned}
$$

and $\mathfrak{R}_{1}, \mathfrak{R}_{2}, \mathfrak{R}_{3}$ and $\mathfrak{R}_{4}$ are smooth functions of arc-length and time.

Proof. Differentiating Eq. (17) with respect to $s$, we have

$$
\begin{aligned}
& \frac{\partial}{\partial s} \frac{\partial \xi_{1}}{\partial t}=\left[\frac{\partial f}{\partial s}+f k_{1}-g\right] T \\
+ & {\left[\frac{\partial g}{\partial s}+g k_{1}+f\right] \eta } \\
+ & {\left[g k_{2}+h k_{3}\right] \xi_{1} } \\
+ & {\left[\frac{\partial h}{\partial s}+h k_{1}\right] \xi_{2} . }
\end{aligned}
$$

Under the assumption of spacelike curve $\zeta$, we can easily obtain that 


$$
\begin{aligned}
& \frac{\partial}{\partial t} \frac{\partial \xi_{1}}{\partial s}=\left[k_{2}\left(\frac{\partial \Re_{2}}{\partial s}+\Re_{1}+\Re_{2} k_{1}-\Re_{3} k_{2}\right)\right. \\
& -k_{2}\left[\frac{\partial}{\partial s}\left(\frac{\partial \Re_{2}}{\partial s}+\Re_{1}+\Re_{2} k_{1}-\Re_{3} k_{2}\right)\right. \\
& \left.+f k_{1}\right] T \\
& +\left[( k _ { 2 } ^ { 2 } - 1 ) \left(\frac{\partial \Re_{3}}{\partial s}+\mathfrak{R}_{2} k_{2}\right.\right. \\
& +k_{2}\left(\frac{\partial \Re_{2}}{\partial s}+\Re_{1}+\mathfrak{R}_{2} k_{1}-\mathfrak{R}_{3} k_{2}\right) \\
& \left.+k_{3}\left(\frac{\partial \Re_{4}}{\partial s}+\Re_{3} k_{3}+\Re_{4} k_{1}\right)\right] \xi_{1} \\
& \left.+\mathfrak{R}_{3} k_{1}+\mathfrak{R}_{4} k_{3}\right) \\
& -k_{2} \frac{\partial}{\partial s}\left(\frac{\partial \Re_{2}}{\partial s}+\Re_{1}+\mathfrak{R}_{2} k_{1}\right. \\
& \left.-\mathfrak{R}_{3} k_{2}\right) \\
& -\frac{\partial}{\partial s}\left[\frac { \partial } { \partial s } \left(\frac{\partial \Re_{3}}{\partial s}+\Re_{2} k_{2}+\Re_{3} k_{1}\right.\right. \\
& +\left[\frac{\partial}{\partial s}\left(\frac{\partial \mathfrak{R}_{4}}{\partial s}+\mathfrak{R}_{3} k_{3}+\mathfrak{R}_{4} k_{1}\right)\right. \\
& +k_{3}\left(\frac{\partial \mathfrak{R}_{3}}{\partial s}+\mathfrak{R}_{2} k_{2}+\mathfrak{R}_{3} k_{1}+\mathfrak{R}_{4} k_{3}\right)+\frac{\partial k_{3}}{\partial t} \\
& \left.+h k_{1}\right] \xi_{2}+k_{3} \frac{\partial \xi_{2}}{\partial t} \text {. } \\
& \left.+\mathfrak{R}_{4} k_{3}\right) \\
& +k_{2}\left(\frac{\partial \Re_{2}}{\partial s}+\Re_{1}+\mathfrak{R}_{2} k_{1}\right. \\
& \left.-\Re_{3} k_{2}\right) \\
& \left.+k_{3}\left(\frac{\partial \Re_{4}}{\partial s}+\mathfrak{R}_{3} k_{3}+\mathfrak{R}_{4} k_{1}\right)\right] \\
& -k_{1}\left[\frac { \partial } { \partial s } \left(\frac{\partial \mathfrak{R}_{3}}{\partial s}+\mathfrak{R}_{2} k_{2}+\mathfrak{R}_{3} k_{1}\right.\right. \\
& \left.+\Re_{4} k_{3}\right) \\
& +\frac{\partial}{\partial s}\left(\frac{\partial \Re_{2}}{\partial s}+\Re_{1}+\Re_{2} k_{1}\right. \\
& \left.\left.-\mathfrak{R}_{3} k_{2}\right)\right] \\
& -k_{3}\left[\frac{\partial}{\partial s}\left(\frac{\partial \Re_{4}}{\partial s}+\mathfrak{R}_{3} k_{3}+\mathfrak{R}_{4} k_{1}\right)\right. \\
& +k_{3}\left(\frac{\partial \Re_{3}}{\partial s}+\Re_{2} k_{2}+\mathfrak{R}_{3} k_{1}\right. \\
& \left.\left.\left.+\mathfrak{R}_{4} k_{3}\right)\right]+g k_{1}\right] \eta \\
& k_{3} \frac{\partial \xi_{2}}{\partial t}=\left[\frac{\partial f}{\partial s}-k_{2}\left(\frac{\partial \Re_{2}}{\partial s}+\Re_{1}+\Re_{2} k_{1}\right.\right. \\
& \left.\left.-\mathfrak{R}_{3} k_{2}\right)-g\right] T
\end{aligned}
$$




$$
\begin{aligned}
& +\left[\frac{\partial g}{\partial s}-\left(k_{2}+\frac{2}{k_{2}}\right) \frac{\partial}{\partial s}\left(\frac{\partial \Re_{2}}{\partial s}+\Re_{1}+\mathfrak{R}_{2} k_{1}\right.\right. \\
& \left.-\mathfrak{R}_{3} k_{2}\right) \\
& +\left(k_{2}^{2}-1\right)\left(\frac{\partial \Re_{3}}{\partial s}+\Re_{2} k_{2}\right. \\
& \left.+\mathfrak{R}_{3} k_{1}+\mathfrak{R}_{4} k_{3}\right) \\
& -\frac{\partial}{\partial s}\left[\frac { \partial } { \partial s } \left(\frac{\partial \Re_{3}}{\partial s}+\mathfrak{R}_{2} k_{2}+\mathfrak{R}_{3} k_{1}\right.\right. \\
& \left.+\mathfrak{R}_{4} k_{3}\right) \\
& +k_{2}\left(\frac{\partial \Re_{2}}{\partial s}+\Re_{1}+\Re_{2} k_{1}\right. \\
& \left.-\mathfrak{R}_{3} k_{2}\right) \\
& \left.+k_{3}\left(\frac{\partial \mathfrak{R}_{4}}{\partial s}+\mathfrak{R}_{3} k_{3}+\mathfrak{R}_{4} k_{1}\right)\right] \\
& -k_{1}\left[\frac { \partial } { \partial s } \left(\frac{\partial \Re_{3}}{\partial s}+\mathfrak{R}_{2} k_{2}+\mathfrak{R}_{3} k_{1}\right.\right. \\
& \left.+\mathfrak{R}_{4} k_{3}\right) \\
& +\frac{\partial}{\partial s}\left(\frac{\partial \Re_{2}}{\partial s}+\Re_{1}+\Re_{2} k_{1}\right. \\
& \left.\left.-\Re_{3} k_{2}\right)\right] \\
& -k_{3}\left[\frac{\partial}{\partial s}\left(\frac{\partial \Re_{4}}{\partial s}+\mathfrak{R}_{3} k_{3}+\mathfrak{R}_{4} k_{1}\right)\right. \\
& +k_{3}\left(\frac{\partial \Re_{3}}{\partial s}+\Re_{2} k_{2}+\Re_{3} k_{1}\right. \\
& \left.\left.\left.+\mathfrak{R}_{4} k_{3}\right)\right]\right] \eta
\end{aligned}
$$$$
\begin{aligned}
& +\left[h k_{3}\right. \\
& +k_{2}\left[\frac{\partial}{\partial s}\left(\frac{\partial \mathfrak{R}_{2}}{\partial s}+\mathfrak{R}_{1}+\mathfrak{R}_{2} k_{1}-\mathfrak{R}_{3} k_{2}\right)\right. \\
& +k_{1}\left(\frac{\partial \mathfrak{R}_{2}}{\partial s}+\mathfrak{R}_{1}+\mathfrak{R}_{2} k_{1}-\mathfrak{R}_{3} k_{2}\right) \\
& \left.\left.+k_{3}\left(\frac{\partial \mathfrak{R}_{4}}{\partial s}+\mathfrak{R}_{3} k_{3}+\mathfrak{R}_{4} k_{1}\right)+g\right]\right] \xi_{1} \\
& +\left[\frac{\partial h}{\partial s}-\frac{\partial}{\partial s}\left(\frac{\partial \mathfrak{R}_{4}}{\partial s}+\mathfrak{R}_{3} k_{3}+\mathfrak{R}_{4} k_{1}\right)\right. \\
& -k_{3}\left(\frac{\partial \mathfrak{R}_{3}}{\partial s}+\mathfrak{R}_{2} k_{2}+\mathfrak{R}_{3} k_{1}+\mathfrak{R}_{4} k_{3}\right) \\
& \left.-\frac{\partial k_{3}}{\partial t}\right] \xi_{2} .
\end{aligned}
$$

Since

$$
\mathfrak{F}\left(\xi_{2}, \frac{\partial \xi_{2}}{\partial t}\right)=0
$$

Hence, the proof is complete.

Lemma 3.3 let $\frac{\partial \zeta}{\partial t}$ be a smooth inextensible flow of the spacelike curve $\zeta$ according to equiform frame in 4-dimensional Minkowski space $\mathbb{R}_{1}^{4}$. The evolution of $k_{3}$ satisfy the partial differential equation 


$$
\begin{aligned}
& \frac{\partial k_{3}}{\partial t} \\
& =\frac{\partial}{\partial s}\left[\frac { 1 } { k _ { 2 } } \left[k _ { 3 } \left[\frac { \partial } { \partial s } \left(\frac{\partial \Re_{3}}{\partial s}+\mathfrak{R}_{2} k_{2}+\mathfrak{R}_{3} k_{1}\right.\right.\right.\right. \\
& \left.+\mathfrak{R}_{4} k_{3}\right)+k_{2}\left(\frac{\partial \Re_{2}}{\partial s}+\Re_{1}+\Re_{2} k_{1}-\mathfrak{R}_{3} k_{2}\right) \\
& \left.+k_{3}\left(\frac{\partial \mathfrak{R}_{4}}{\partial s}+\mathfrak{R}_{3} k_{3}+\mathfrak{R}_{4} k_{1}\right)\right] \\
& +\frac{\partial}{\partial s}\left[\frac{\partial}{\partial s}\left(\frac{\partial \Re_{4}}{\partial s}+\mathfrak{R}_{3} k_{3}+\mathfrak{R}_{4} k_{1}\right)\right. \\
& \left.+k_{3}\left(\frac{\partial \mathfrak{R}_{3}}{\partial s}+\mathfrak{R}_{2} k_{2}+\mathfrak{R}_{3} k_{1}+\mathfrak{R}_{4} k_{3}\right)\right] \\
& \left.\left.-\left(\frac{\partial \mathfrak{R}_{4}}{\partial s}+\mathfrak{R}_{3} k_{3}+\mathfrak{R}_{4} k_{1}\right)\right]\right] \\
& -\frac{\partial}{\partial s}\left(\frac{\partial \mathfrak{R}_{4}}{\partial s}+\mathfrak{R}_{3} k_{3}+\mathfrak{R}_{4} k_{1}\right) \\
& -k_{3}\left(\frac{\partial \mathfrak{R}_{3}}{\partial s}+\mathfrak{R}_{2} k_{2}+\mathfrak{R}_{3} k_{1}\right. \\
& \left.+\mathfrak{R}_{4} k_{3}\right) .
\end{aligned}
$$

Proof. It is obvious directly from theorem 3.5. This complete the proof.

\section{Application of inextensible flows of spacelike curves in $\mathbb{R}_{1}^{4}$}

In this section, we give an application of a special case of inextensible flows of spacelike curves in $\mathbb{R}_{1}^{4}$. Let $\mathfrak{R}_{1}=$ constant $=a \neq 0, \mathfrak{R} \_2$ $=\mathfrak{R}_{3}=0$ and $\mathfrak{R}_{4}=\frac{a}{k_{1}(s, t)}$. From Eqs. (12), (21) and (23), we have the PDE system

$$
\frac{\partial k_{1}}{\partial t}=0
$$

$$
\begin{aligned}
& \frac{\partial k_{2}}{\partial t}=a\left(\frac{2 k_{3}+1}{k_{1}^{3}}\right)\left[2\left(\frac{\partial k_{1}}{\partial s}\right)^{2}-k_{1}\left(\frac{\partial^{2} k_{1}}{\partial s^{2}}\right)\right] \\
& +\left(\frac{a}{k_{1}}\right)\left[\left(\frac{\partial^{2} k_{3}}{\partial s^{2}}\right)\right. \\
& \left.+k_{3}\left[\left(\frac{\partial k_{1}}{\partial s}\right)+k_{2}^{2}\right]\right] \\
& -\left(\frac{3 a}{k_{1}^{2}}\right)\left(\frac{\partial k_{1}}{\partial s}\right)\left(\frac{\partial k_{3}}{\partial s}\right) \text {, } \\
& \frac{\partial k_{3}}{\partial t} \\
& =a \frac{\partial}{\partial s}\left\{( \frac { k _ { 3 } } { k _ { 2 } } ) \left[\frac{\partial}{\partial s}\left(\frac{k_{3}}{k_{1}}\right)+k_{3}\left[\frac{\partial}{\partial s}\left(\frac{1}{k_{1}}\right)+1\right]\right.\right. \\
& \left.+k_{2}\right]+\left(\frac{1}{k_{2}}\right) \frac{\partial}{\partial s}\left[\frac{\partial^{2}}{\partial s^{2}}\left(\frac{1}{k_{1}}\right)+\left(\frac{k_{3}^{2}}{k_{1}}\right)\right] \\
& \left.-\left(\frac{1}{k_{2}}\right)\left[\frac{\partial}{\partial s}\left(\frac{1}{k_{1}}\right)+1\right]\right\}-a \frac{\partial^{2}}{\partial s^{2}}\left(\frac{1}{k_{1}}\right) \\
& -\frac{a k_{3}^{2}}{k_{1}} \text {. }
\end{aligned}
$$

By solving the system (27) numerically, one solution of this system is

$$
\begin{gathered}
k_{1}(s, t)=c_{1}, \\
k_{2}(s, t)=\frac{a}{c_{2} c_{3}}\left[1-\tanh \left(c_{2} s+c_{3} t\right)\right], \\
k_{3}(s, t)=c_{2}^{2}\left(c_{3}-a\right) \tanh ^{2}\left(c_{2} s+c_{3} t\right),
\end{gathered}
$$

where $c_{1}, c_{2}$ and $c_{3}$ are constants such that $c_{2}, c_{3} \neq 0$. The curvatures of the family of spacelike curves $C_{t}$ as a function of the coordinates $s$ and $t$ are plotted in Figure 1, Figure 2 and Figure 3. 


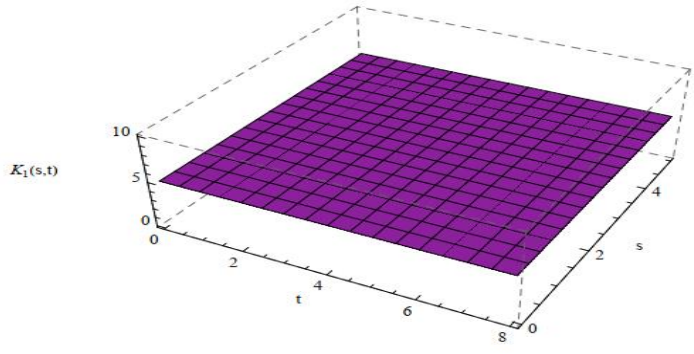

Fig.1: The curvature $k_{1}(s, t)$ of the family of spacelike curves $C_{t}$ for $s \in[0,5], t \in[0,8]$ and $c_{1}=0$.

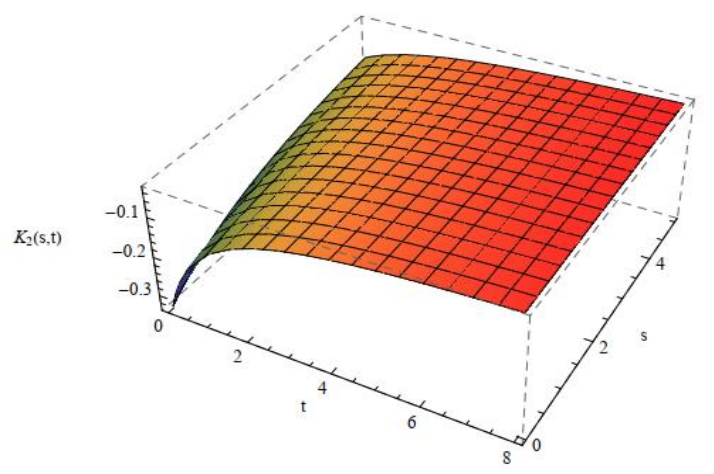

Fig.2: The curvature $k_{2}(s, t)$ of the family of spacelike curves $C_{t}$ for $s \in[0,5], t \in[0,8]$, $a=1, c_{2}=4$ and $c_{3}=2$.

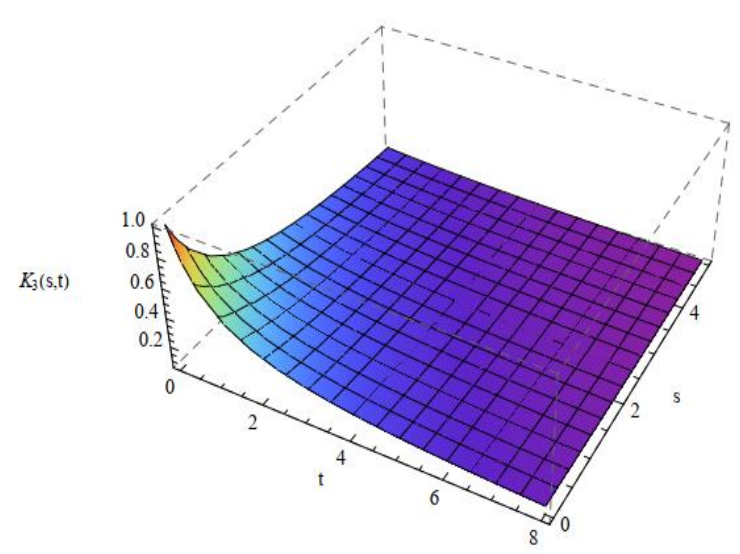

Fig.3: The curvature $k_{3}(s, t)$ of the family of spacelike curves $C_{t}$ for $s \in[0,5], t \in[0,8]$, $a=1, c_{2}=4$ and $c_{3}=2$.

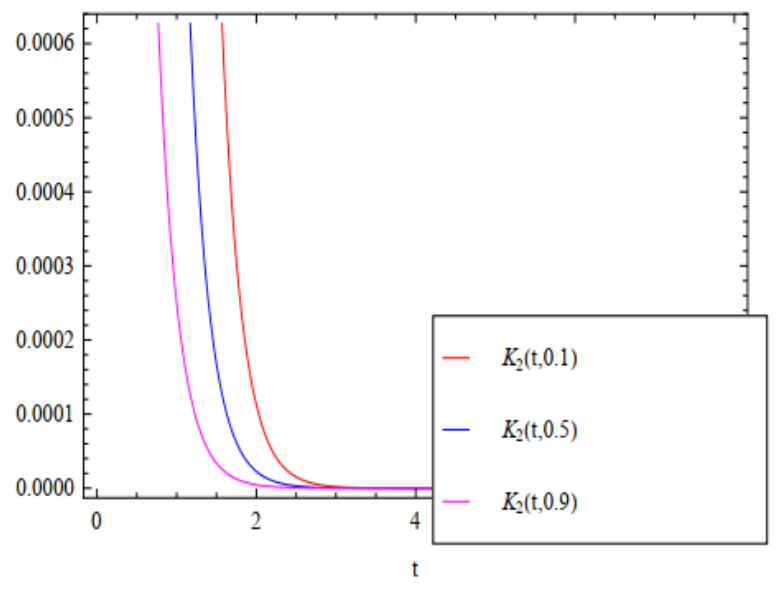

Fig.4: The curvature $k_{2}(s, t)$ for $s=0.1,0.5$ and 0.9 .

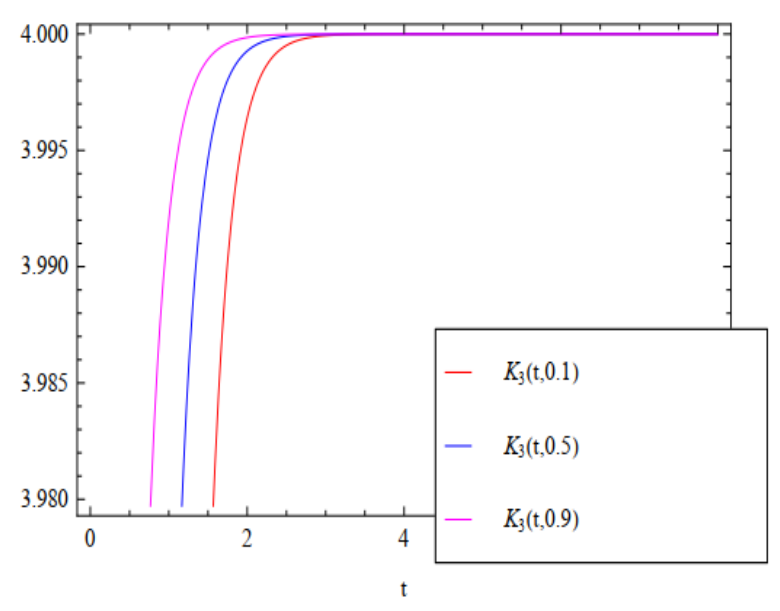

Fig. 5: The curvature $k_{3}(s, t)$ for $s=0.1,0.5$ and 0.9 . 


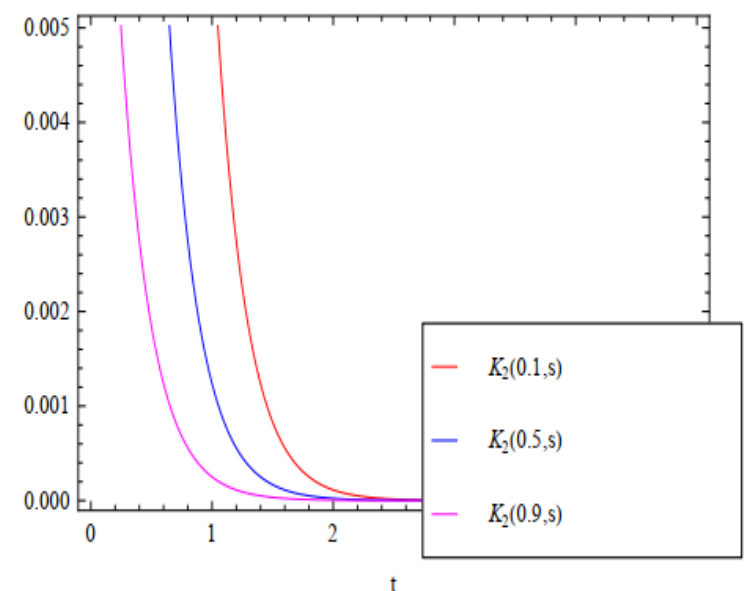

Fig.6: The curvature $k_{2}(s, t)$ for $t=0.1,0.5$ and 0.9

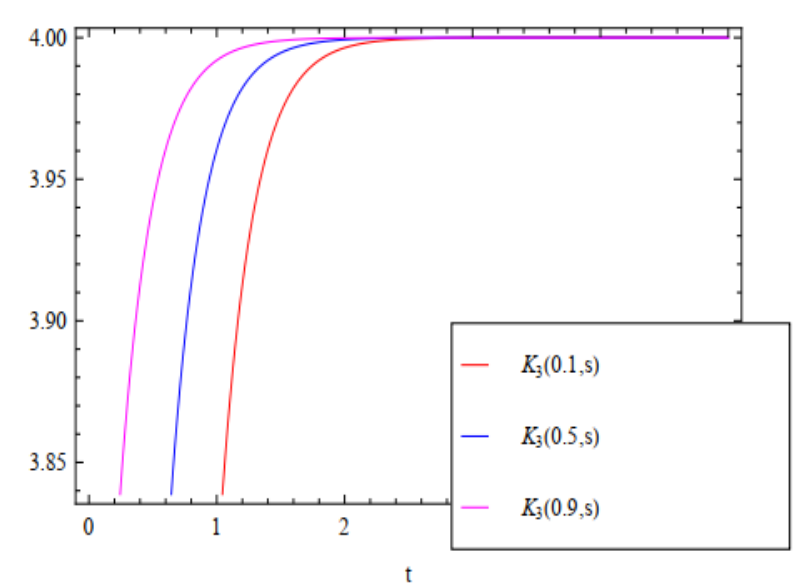

Fig.7: The curvature $k_{3}(s, t)$ for $t=0.1,0.5$

\section{Conclusion}

As a conclusion of our results, the inextensible flows of spacelike curves lying fully on a spacelike surface $\Omega$ according to the equiform frame in 4-dimensional Minkowski space $\mathbb{R}_{1}^{4}$ can be expressed as a partial differential equation involving the equiform curvature functions in $\mathbb{R}_{1}^{4}$. Also, the advancement equations for the curvatures of the curve in terms of these velocities are derived and found its exact solutions.

\section{References:}

[1] S. J. Altschuler, M. A. Grayson, Shortening space curves and flow through singularities, J. Differ. Geom., 33 (1992), 283--298.
[2] H. S. Abdel-Aziz, M. Khalifa Saad and A. A. Abdel-Salam, Equiform Differential Geometry of curves in Minkowski spacetime, arXiv:1501.02283v1 [math.DG] 9 Jan 2015.

[3] D. Conte, B. Paternoster, L. Moradi and F. Mohammadi, Construction of exponentially fitted explicit peer methods, International Journal of Circuits, Systems and Signal Processing, pp. 501-506, Volume 13, 2019.

[4] Yu. K. Demjanovich, T. O. Evdokimova, O. N. Ivancova, D. M. Lebedinskii, A. Y. Ponomareva, On Wavelet Decomposition of the Singular Splines, International Journal of Circuits, Systems and Signal Processing, pp. 571-579, Volume 14, 2020.

[5] M. P. Do Carmo, Differential geometry of curves and surfaces, Prentice Hall, Englewood Cliffs, NJ, 1976.

[6] M. Evren Aydin, MahmutErgut, The equiform differential geometry of curves in 4-dimensional galilean space $G_{4}$, Stud. Univ. Babes-Bolyai Math.,58 (3) (2013), 399--406.

[7] M. Gage, R. S. Hamilton, The heat equation shrinking convex plane curves, J. Differ. Geom., 23 (1986), 69—96

[8] M. Grayson, The heat equation shrinks embedded plane curves to round points, J. Differ. Geom., 26 (1987), 285--314.

[9] H. Gun Bozok, H. Oztekin, Inextensible flows of curves in the equiform geometry of 4-dimensional Galilean space $G_{4}$, Facta Universitais, 30 (2) (2015), 209--216.

[10]R. A. Hussien, S. G. Mohamed, Generated surfaces via inextensible flows of curves in $\mathbb{R}^{3}$, J. of Applied Math.,(2016), Article ID 6178961, 8 pages http://dx.doi.org/10.1155/2016/6178961

[11]D. Y. Kwon, F. C. Park, D. P. Chi, Inextensible flows of curves and develoble surfaces, Applied Math. Letters, 18 (2005), 1156--1162.

[12]T. Korpinar, On Inextensible flows of curves according to alternative moving frame, J. of Dyn. Syst. and Geomet. Theories, 15 (1) (2017), 15--27.

[13] T. Korpinar, New inextensible flows of principal normal spherical image, Asian- 
European Journal of Mathematics, 11 (1) (2018), (14 pages), https://doi.org/10.1142/S179355711850001 8

[14] R. Lopez, Differential Geometry of Curves and Surfaces in Lorentz-Minkowski Space, International Electronic Journal of Geometry, 7 (1) (2014), 44-107

[15] S. G. Mohamed, Binormal motions of inextensible curves in de-Sitter space $S^{2,1}$, J. of the Egy. Math. Soci., 25 (2017), 313-318.

[16]B. O'Neill, Semi-Riemannian geometry with applications to relativity, Academic press, New York, 1983.

[17] S. Yilmaz, M. Turgut, On the Differential Geometry of the curves in Minkowski space-time, Int. J. Contemp. Math. Sci., 3 (7) (2008), 1343--1349.

[18] O. G. Yildiz, S. Ersoy, M. Masal, A note on inextensible flows of curvature on oriented surface, CUBO A Math. J., 16 (3) (2014), 11--19.

[19] M. Yeneroglu, On new characterization of inextensible flows of pace-like curves in de Sitter space, Open Math., 14 (2016), 946-954.

\section{Acknowledgments}

The authors would like to express their deep thanks to the referee for his/her careful reading and many valuable suggestions towards the improvement of the paper.

\section{Authors contributions}

All the authors have made substantive contributions to the article and assume full responsibility for its content. Both authors read and approved the final manuscript.

\section{Funding}

No fund in conducting this research.

\section{Availability of data and materials}

All the data and materials used in this paper can be freely available.

Competing interests

The authors declare that they have no competing interests.

\section{Creative Commons Attribution License 4.0 (Attribution 4.0 International, CC BY 4.0)}

This article is published under the terms of the Creative Commons Attribution License 4.0

https://creativecommons.org/licenses/by/4.0/deed.en_US 